\title{
Delayed-onset ureteral lesions due to thermal energy: An emerging condition
}

\author{
Cesare Selli, Filippo Maria Turri, Cristina Gabellieri, Francesca Manassero, Maurizio De Maria, \\ Andrea Mogorovich \\ Department of Urology, University of Pisa, Italy.
}

\begin{abstract}
Summary Objectives: To describe the risks of ureteral damage occurring during urological and gynecological procedures utilizing energybased surgical devices (ESD) during both laparoscopic and open procedures.

Materials and Methods: During the last 20 months we observed five cases of iatrogenic ureteral lesions caused by ESD which required open surgery. There were 3 lesions of the lower ureter occurring during gynecological laparoscopic or robotic procedures, and 2 lesions of the upper ureter occurring during open enucleation of low-stage renal cell carcinomas.

Results: In the laparoscopic gynecological lesions the cause was attributable to monopolar cutting and bipolar coagulation: they presented with urine extravasation after 20, 15 and 15 days respectively and required ureteral reimplantation in 2 out of 3 cases. In the upper ureteral lesions the causes were bipolar coagulation and LigaSure Impact TM used for perirenal fat dissection: they presented after 2 and 4 months respectively and required uretero-ureterostomy and inferior nephropexy in one case and nephrectomy in the other. In 3 out of 5 cases there was an unsuccessful attempt at placing an ureteral double J stent, and in the 2 cases where it was placed it did not prevent the formation of subsequent stricture in one.

Conclusions: The widespread diffusion of ESD has the potential drawback of inadvertent thermal energy transmission to the ureter. Delayed presentation of ureteral lesions and difficulties in ureteral stent placement were the common features of the cases observed. Inadvertent ureteral damage by different thermal energy sources is an emerging condition, requiring awareness, prompt recognition and adequate treatment with the reconstructive urology principles.
\end{abstract}

KEY WORDS: Ureteral injury; Energy-based surgical devices; Reconstructive urology.

Submitted 24 October 2013; Accepted 31 January 2014

\section{AIM}

Energy-based surgical devices (ESD) are increasingly used both in laparoscopic and open procedures in many surgical branches The most frequently used systems are electrosurgical (monopolar, bipolar, impedance-con- trolled bipolar) and ultrasonic (1-3). The ureter, due to its relatively small caliber and continuous flow of urine is particularly susceptible to inadvertent damage with thermal energy: monopolar energy is the most destructive one and laparoscopic lesions tend to be more extensive than those occurring in open surgery (4-5).

\section{Case report}

We report herein our experience with the management of inadvertent ureteral lesions due to ESD occurring during both open and laparoscopic gynecological and urological procedures.

Cases Report are described in Supplementary materials posted on www.aiua.it

\section{Discussion}

In a review of laparoscopic ureteral injury in pelvic surgery, Ostrenski et al. (6) in 2003 reported a delayed clinical occurrence in $70 \%$ of cases, with thermal injury accounting for only $1.4 \%$. However with the present spread of minimally invasive gynecologic surgery and the use of ESD also in open procedures, this percentage is presumably higher.

In laparoscopic gynecological procedures the ureter is likely to be injured in three locations: at the infundibolopelvic ligament, deep to the ovarian fossa and at the ureteral canal (6). In the three cases that came at our attention the site of thermal damage was likely to be of the third type.

A constant care of the ureteral location should characterize all female pelvic dissections, and this applies particularly to robotic surgery, where the tri-dimensional vision and the possibility of a very fine dissection, possibly minimizing the amount of thermal energy, are intrinsic advantages of the technology.

Thermal injury of the upper ureter has been reported following percutaneous radiofrequency treatment of small renal tumors (7) or as a consequence of laparoscopic partial nephrectomy using a microwave tissue coagulator (8), while to the best of our knowledge there is no description of ureteral damage caused by ESD for dissection of the perirenal fat during open conservative surgery for kidney tumors. 
In our experience, as well as in that of others, ureteral lesions secondary to ESD present a delayed clinical appearance, in common with other iatrogenic damages (9-11). This can be explained by the fact that thermal injury damages vascular supply beyond the area of actual contact, leading to delayed necrosis or scarring of the ureteral wall (11). The real extent of tissue damage is greater than that appearing with imaging techniques, and adequate ureteral mobilization is necessary for tensionfree surgical repair.

In the present experience upper ureteral lesions became clinically evident later than lower ureteral lesions associated with gynecological procedures, which all became apparent between two and three post-operative weeks with the appearance of either vaginal discharge or with acute abdomen due to uroperitoneum.

An endourological approach should be obviously attempted first, but in our experience in 3 out of 5 cases the placement of a double J stent was unsuccessful and in one out of 2 case when it was placed it did not prevent the formation of subsequent stricture.

We believe that, in case of failure of conservative management, an open surgical approach provides the best chances for and adequate ureteral mobilization, necessary for tension-free surgical repair, but robot-assisted

\section{Figure 1.}

Case 1. Uro-CT scan demonstrating uroperitoneum and a jet of contrast medium originating from the right pelvic ureter.

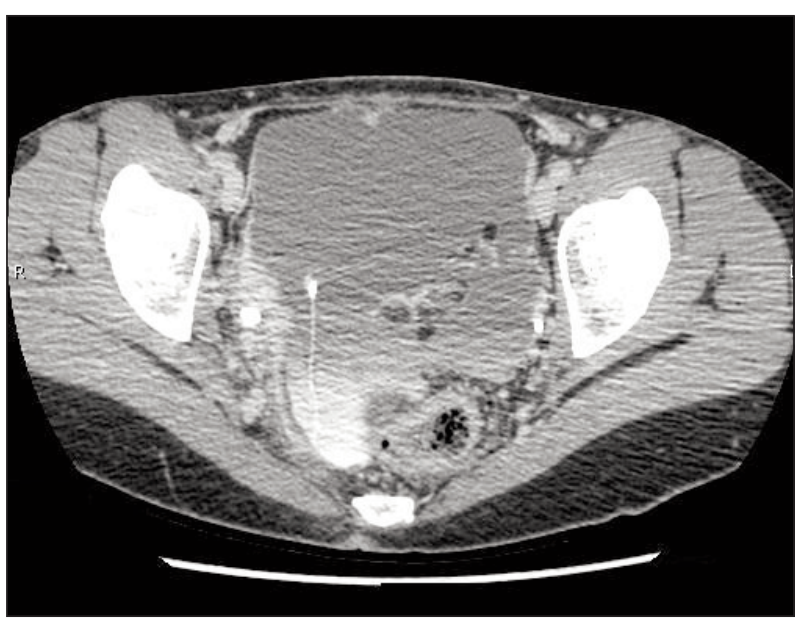

ureteral reconstructive procedures are showing promising results (12).

Figures are reported in Supplementary materials posted on www.aiua.it

\section{REFERENCES}

1. Song C, Tang B, Campbell PA, et al. Thermal spread and heat absorbance differences between open and laparoscopic surgeries during energized dissections by electrosurgical instruments. Surg Endosc. 2009; 23:2480-2487.

2. Alkatout I, Schollmeyert T, Haldawar N, et al. Principles and safety measures of electrosurgery in laparoscopy. J Soc Lap Surg. 2012; 16:130-139.

3. Kennedy JS, Stranahan PL, Taylor KD, Chandler JG: High burststrength feedback-controlled bipolar vessel sealing. Surg Endosc. 1998; 12:876-878.

4. Phillips CK, Hruby GW, Durak E, et al. Tissue response to surgical energy devices. Urology. 2008; 71:744-748.

5. Tulikangas PK, Smith T, Falcone T, et al. Gross and histologic characteristics of laparoscopic injuries with four different energy sources. Fertil Steril. 2001; 75:806-810.

6. Ostrzenski A, Radolinski B, Ostrzenska KM. A review of laparoscopic ureteral injury in pelvic surgery. Obstet Gynecol Surv. 2003; 58:794-799.

7. Doody O, Given MF, Harper M, et al. Rendezvous technique following thermal ureteric injury after radiofrequency ablation in a solitary kidney. J Vasc Interv Radiol. 2008; 19:1112-1114.

8. Harabayashi T, Shinohara N, Kakizaki H, et al. Ureteral stricture developing after partial nephrectomy with a microwave tissue coagulator: case report. J Endourol. 2003; 17:919-921.

9. Manoucheri E, Cohen SL, Sandberg EM, et al. Ureteral injury in laparoscopic gynecologic surgery. Rev Obstet Gynecol. 2012; 5:106-111.

10. Gao JS, Leng JH, Liu ZF, et al. Ureteral injury during gynecological laparoscopic surgeries: report of twelve cases. Chin Med Sci J. 2007; 22:13-16.

11. Oh BR, Kwon DD, Park KS, et al. Late presentation of ureteral injury after laparoscopic surgery. Obstet Gynecol. 2000; 95:337-339.

12. Kozinn SI, Canes D, Sorcini A, et al. Robotic versus open distal ureteral reconstruction and reimplantation for benign stricture disease. J Endourol. 2012; 26:147-151.

\footnotetext{
Correspondence

Cesare Selli, MD (Corresponding Author)

c.selli@med.unipi.it

Filippo Maria Turri, MD

fm.turri@gmail.com

Cristina Gabellieri, MD

gabelliericristina@interfree.it

Francesca Manassero, MD

francy.manassero@hotmail.com

Maurizio De Maria, MD

m.demaria@ao-pisa.toscana.it

Andrea Mogorovich, MD

mogorovich@hotmail.it

Urologia Universitaria - Via Paradisa 2 - 50124 Pisa, Italy
} 